\title{
Bisphosphonates in Postmenopausal Women with Osteoporosis to Prevent Future Fractures
}

\section{Bashir I* and Rahman Z}

The Department of Physical Medicine and Rehabilitation, Jackson park hospital, USA

*Corresponding author: Bashir Imam, The Department of Physical Medicine and Rehabilitation, Jackson park hospital, 7531 Stony Island Avenue Chicago, Illinois 60649, USA, Tel: 204869 5003; Email: bashir_imam@hotmail.com

\section{Review Article}

Volume 3 Issue 2

Received Date: April 01, 2019

Published Date: April 24, 2019

DOI: $10.23880 /$ jobd-16000179

\section{Abstract}

Postmenopausal women who have osteoporosis are at increased risk of future fractures. Bisphosphonates are drugs that are used to treat osteoporosis by acting on the osteoclasts to inhibit bone resorption. Several studies have shown that bisphosphonates can maintain or even increase bone mineral density in osteoporosis patients. This review study analyzed the literature on clinical experiments with bisphosphonate therapy in postmenopausal women to determine if these drugs are efficacious in preventing future fractures. Four out of five studies found that women treated with bisphosphonates were at decreased risk of future fractures, and six of six studies found that bisphosphonate therapy increases bone mineral density relative to placebo control. Although further work is warranted to understand the level of bone mineral density increase that is associated with fracture prevention, this study implies that bisphosphonate therapy can be used to help prevent future fractures in postmenopausal osteoporotic women. The study is significant in that it helps to underscore the efficacy of bisphosphonate therapy in postmenopausal women, and it may be generalizable to other populations with osteoposrosis who are at increased risk of fractures.

Keywords: Bisphosphonate; Postmenopausal Osteoporosis; Fracture Risk Reduction; Side Effects

\section{Introduction}

Postmenopausal women are at increased risk of osteoporosis, and an estimated 40 million women in the United States are estimated to have low bone mineral density [1]. Such loss of bone mineral density and the resultant deterioration of bone architecture are known to lead to increased risk of fractures [2]. Fractures of the hip and spine are the most common that occur as a result of osteoporosis, and they can lead to reduced quality of life, dependent living situations, and increased risk of death, in addition to psychological problems such as lowered selfesteem [1].

Bisphosphonates are drugs that are used to treat osteoporosis. They block the action of the bone cells known as osteoclasts and thereby inhibit the resorption of the bone itself [3]. Bisphosphonates are the first line of defense against osteoporosis, and can decrease the rate of bone turnover, increase bone mineral density, and may thereby reduce the risk of fractures in patients with osteoporosis [4]. Because postmenopausal women are at increased risk of osteoporosis and resulting fractures, it 


\section{Journal of Orthopedics \& Bone Disorders}

stands to reason that bisphosphonates might be used in this group to prevent future fractures.

The purpose of the present paper is to review the literature on therapy in postmenopausal women to determine if these drugs are efficacious in preventing future fractures. Specifically, the goal is to determine if there is clinical evidence that supports the use of bisphosphonates to reduce the risk of future fractures. The hypothesis of interest is that postmenopausal women who receive bisphosphonate treatment will have a reduced fracture risk relative to women who do not receive the treatment. The reduced fracture risk was measured by assessing the number of subsequent fractures and/or by assessing the bone mineral density following bisphosphonate therapy.

\section{Materials and Methods}

A keyword search strategy was used to search databases to locate abstracts for the study. The databases PubMed and ClinicalTrials.gov were searched using combinations of the key words "osteoporosis," "fracture," "post-menopause," and "bisphosphonate." The parameters "human" and "English language" was selected. All bisphosphonates were included, administered either orally or intravenously, according to the established protocols for administration and without co-medications, in postmenopausal women ages 65 or older who are at risk, including those with comorbidities, throughout the entire world. Inclusion criteria for journals included those that are respectable, publishing peer-reviewed articles with clearly presented methodology sections; follow ups of more than one year and in which primary outcomes were the number of fractures of any localization and/or bone density and secondary outcomes were side effects. All dates up to the present time were searched.

Randomized clinical trials with a follow-up period of at least one year were chosen; trials without randomization or without at least one year follow-up were excluded. Studies that are not randomized controlled trials or systematic reviews of such were excluded.

Studies were chosen with outcome data that included either fracture incidence after at least a 1 year follow-up period, bone mineral density after at least a 1 year follow up period, or both. Secondary outcomes were the side effects. Because a limited number of articles with all of these criteria were found, and because the data were not entirely comparable across all located studies, statistical analyses were not performed. We screened the titles and abstracts to select the articles for full-text review. The articles were evaluated using the CEBM appraisal worksheets.

\section{Results}

Using the PRISMA 2009 Flow diagram, the search for osteoporosis rendered 73,809 records, that for bisphosphonates resulted in 25,382 records, the search for post-menopause gave 24,741 records, and the search for fractures rendered 204,531 records. This was a total of 328,463 , but some of these were duplicates and very few combined all four subjects appropriately. After duplicating articles were removed, the search left 143 records. All of the abstracts for these 143 records were examined, and there were a total of 8 studies that involved postmenopausal women ages 65 or older who had osteoporosis and who were part of a randomized, placebo-controlled clinical trial. Outcomes assessed for these trials involved fractures after at least a 1 year follow-up period and/or a comparison of change in bone mineral density relative to controls. All eight of these studies were included in the final analyses. Only five of the trials reported fracture data, six of the trials reported bone mineral density data, and all of the trials reported side effects. No quantitative synthesis was performed because the reporting of the data was not consistent across all studies.

All of the included search parameters together turned up 8 studies that involved postmenopausal women ages 65 or older who had osteoporosis and who were part of a randomized, placebo-controlled clinical trial. Outcomes assessed for these trials involved fractures after at least a 1 year follow-up period and/or a comparison of change in bone mineral density relative to controls. Only five of the trials reported fracture data, six of the trials reported bone mineral density data, and three of the trials reported side effects. The results of the study are presented in Tabular form in Table 1, and are described below.

\section{Fractures}

Five studies reported data on fractures [5-9]. Osaki, et al. [5] performed a study on 529 postmenopausal women and found that 5 out of 173 in the treatment group $(2.89 \%)$ and 32 out of 356 in the control group (8.99\%) suffered fractures-the authors reported that this was a statistically significant difference at the $\mathrm{p}=0.01$ level. Jacques, et al. [6] performed a study on 7736 postmenopausal women and assessed the prevalence of new vertebral fractures. They found a total of 308 fractures in 2976 women in the control group (10.4\%) 


\section{Journal of Orthopedics \& Bone Disorders}

and a total of 23 fractures in 3195 women in the treatment group $(0.7 \%)$, and reported that this was a significant difference at $\mathrm{p}<0.001$. Greenspan, et al. [7] had the opposite findings, in that 17 of $89(20 \%)$ in the treatment group and 14 of $92(16 \%)$ in the placebo control group had vertebral fractures.

Together, these data indicate that the three studies assessed a total of 6881 elderly postmenopausal osteoporotic women for fracture risk after at least 1 year of taking bisphosphonate therapy. Two of the studies found independently of the other that fracture incidence was significantly less in the bisphosphonate treatment group than in the placebo control group, but the third found the opposite [5-7]. If the data for these studies are taken together, then 357 out of 3421 control participants suffered a fracture and 42 out of 3460 treatment participants suffered a fracture, for incidence frequencies of $10.4 \%$ and $1.2 \%$, respectively.

A fourth study also reported data on fracture rates; however, it did not report them per participant, but rather in fracture rates per 100 patient-years [8]. The authors examined 66 postmenopausal osteoporotic women who took the bisphosphonate etidronate. During the period of the study from 60 to 150 weeks, the authors found that there was a statistically greater number of fractures per 100 patient years in the placebo group (54/100 patient years) relative to the treatment group (6/100 patient years). This difference was significant at the $p=0.023$ level.

A fifth study also reported on fracture data, but again the fracture rates were in 100 patient-years units rather than by individual patient. Reid, et al. [9] examined the use of pamidronate in a 2 year study of 48 postmenopausal osteoporotic women. They found that there was a trend for vertebral fracture rates to be lower in the treatment group (13/100 patient years) relative to the placebo group (24/100 patient years), but this was not significant at the $\mathrm{p}<0.05$ level, although it was significant at the $\mathrm{p}<0.1$ level $(\mathrm{p}=0.07)$.

\section{Bone Mineral Density}

Six studies located reported change in bone mineral density over the study period of at least 1 year [7-12 ]. Each of these studies had a slightly different protocol for assessing change in bone mineral density in the control and treatment groups, and each of them had a somewhat different method of reporting their results, so they will not be quantitatively analyzed, but instead reported here individually. Greenspan, et al. [7] conducted a study of
181 women age 65 or older who had osteoporosis. They took either zoledronic acid or placebo in IV form and then hip and spine bone mineral density were assessed after 12 and 24 months [7]. They reported that at 12 and 24 months, bone mineral density changes were greater in the treatment groups by $3.2 \%$ and $3.6 \%$ respectively for the hip, and by $1.8 \%$ and $3.6 \%$ at 12 and 24 months respectively for the spine, and all of these differences were significant at the $\mathrm{p}<0.01$ level.

Grey, et al. [11] evaluated the antiresorptive effects of zoledronate in 180 postmenopausal women. They found that after 2 years there was a significantly greater increase in bone mineral density in the spines and hips of the treatment groups (divided into three groups with different dosages) relative to the placebo group. The changes in bone mineral density in the spines were $4.4 \%$, $5.5 \%$ and $5.3 \%$ for $1 \mathrm{mg}, 2.5 \mathrm{mg}$, and $5 \mathrm{mg}$ zoledronate, respectively. The changes in bone mineral density in the hips were $2.6 \%, 4.4 \%$, and $4.7 \%$ for $1 \mathrm{mg}, 2.5 \mathrm{mg}$, and 5 mg zoledronate, respectively. All of the reported changes were significant at $\mathrm{p}<0.001$.

Popp, et al. [12] analyzed the effects of zoledronate and placebo on the bone mineral density of the spine in 107 postmenopausal women. The study was a three year protocol, and the authors found that there were significantly greater positive changes in bone mineral density in the treatment group relative to the placebo group. At the three year point, there was a $9.58 \%$ increase in bone mineral density of the spine in the treatment group relative to the placebo group, and this change was significant at the $\mathrm{p}<0.0001$ level.

Storm, et al. [8] also examined bone mineral density among 66 postmenopausal women. They found that vertebral bone mineral density significantly increased in the treatment group, while it decreased over the study period in the placebo group. Their results indicated that there was overall a difference of 8.0 percentage points between the two groups at the end of the study. These results were significant at the $\mathrm{p}<0.01$ level.

Adami, et al. [10] studied the bisphosphonate alendronate sodium relative to placebo and to intranasal salmon calcitonin in a 2 year trial of 286 postmenopausal women with osteoporosis. They found that two different doses, 10 and $20 \mathrm{mg}$, of alendronate sodium, increased bone mineral density in the lumbar spine by $4.7 \%$ and $6.1 \%$, respectively, relative to placebo or to intranasal salmon calcitonin. They also examined the femoral neck and trochanter bone mineral density at 10 and $20 \mathrm{mg}$ doses, and found that bone mineral density was increased for the femoral neck by $3.1 \%$ for both doses, and that 


\section{Journal of Orthopedics \& Bone Disorders}

trochanter density was increased by $3.3 \%$ and $3.8 \%$ respectively for the doses.

Reid, et al. [9] examined bone mineral density in the total body, lumbar spine, and femoral trochanter in the 48 postmenopausal women that they studied the effects of pamidronate on. Individuals in the treatment group at all body areas had significantly increased bone mineral density at $1.9 \%$ for the total body, $7 \%$ for the lumbar spine, and $5.4 \%$ for the femoral trochanter. There were no increases seen in bone mineral density for individuals in the placebo group.

\section{Side Effects}

Side effects/adverse events occurred in some studies $[5,7,11]$. Greenspan, et al. [7] reported that $97 \%$ of participants in their trial had an adverse event, and $64 \%$ had a serious adverse event, but there were no statistically significant differences in adverse events between treatment and placebo groups. Side effects reported were cardiac disorders, falling, headache, pyrexia, fatigue, arthralgias, myalgias and flu-like symptoms. Osaki, et al. [5] found that there were adverse events in $20.7 \%$ of the treatment patients and in $21.1 \%$ of the control patients. The most frequent adverse events reported were gastrointestinal disorders in the treatment group, and hip fracture in the control group. Other adverse events included cardiac disorders, death, pneumonia and fracture of the radius, fracture of the spine, dementia, and musculoskeletal and connective tissue disorders. Grey, et al. [11] reported that one participant in the treatment group developed iritis.

\begin{tabular}{|c|c|c|c|c|c|c|c|c|}
\hline \multirow{2}{*}{$\begin{array}{l}\text { Author(s) and } \\
\text { Date }\end{array}$} & \multirow[t]{2}{*}{ Drug(s) } & \multirow{2}{*}{$\begin{array}{c}\text { Admin. } \\
\text { Mode }\end{array}$} & \multicolumn{2}{|c|}{ Fracture (location) } & \multicolumn{2}{|c|}{$\begin{array}{l}\text { Bone Mineral Density (dose } \\
\text { and/or location) }\end{array}$} & \multirow[t]{2}{*}{ Side Effects } & \multirow{2}{*}{$\begin{array}{c}\text { Risks (if } \\
\text { addressed) }\end{array}$} \\
\hline & & & Control & Experimental & Control & Experimental & & \\
\hline Adami, et al. [11] & Alendronate & Oral & & & $\begin{array}{c}4.7 \% / 3.1 \% \\
(10 \\
\mathrm{mg} / 20 \mathrm{mg})\end{array}$ & $\begin{array}{c}6.1 \% / 3.8 \%(10 \\
\mathrm{mg} / 20 \mathrm{mg})\end{array}$ & & \\
\hline $\begin{array}{c}\text { Greenspan, et al. } \\
{[7]}\end{array}$ & Zoledronic acid & I.V. & $\begin{array}{c}16 \% \\
\text { (vertebral) }\end{array}$ & $\begin{array}{c}20 \% \\
\text { (vertebral) }\end{array}$ & $-/-$ & $\begin{array}{l}3.6 \% / 3.6 \% \text { (hip, } \\
\text { spine) }\end{array}$ & \begin{tabular}{|c|} 
Cardiac disorders, \\
falls, headache, \\
pyrexia, fatigue, \\
arthralgias, myalgias, \\
flu-like
\end{tabular} & Falls \\
\hline Grey, et al. [11] & Zoledronate & I.V. & & & $\begin{array}{l}\text {-/-/- (spine) } \\
\text { /-/- (hip) }\end{array}$ & \begin{tabular}{|c|}
$4.4 \% / 5.5 \% / 5.3 \%$ \\
$(1 \mathrm{mg} / 2.5 \mathrm{mg} / 5 \mathrm{mg}$ \\
spine $)$ \\
$2.6 \% / 4.4 \% / 4.7 \%$ \\
$\left(\begin{array}{c}1 \mathrm{mg} / 2.5 \mathrm{mg} / 5 \mathrm{mg} \\
\text { hip })\end{array}\right.$
\end{tabular} & iritis & \\
\hline Jacques, et al. [6] & Zoledronic acid & I.V. & $\begin{array}{c}10.4 \% \\
\text { (vertebral) }\end{array}$ & $\begin{array}{c}0.7 \% \\
\text { (vertebral) }\end{array}$ & & & & \\
\hline Osaki, et al. [5] & Risedronate & Oral & 8.99\% (hip) & 2.89\% (hip) & & & \begin{tabular}{|c|} 
GI disorders, fractures \\
cardiac disorders, \\
death, pneumonia, \\
dementia, \\
musculoskeletal \& \\
connective tissue \\
disorders
\end{tabular} & GI disorders \\
\hline Popp, et al. [12] & Zoledronate & I.V. & & & -(spine) & $8 \%$ (spine) & & \\
\hline Reid, et al. [9] & Pamidronate & Oral & \begin{tabular}{|c|}
$24 / 100$ \\
patient years \\
(vertebral)
\end{tabular} & $\left\{\begin{array}{c}13 / 100 \text { patient } \\
\text { years } \\
\text { (vertebral) }\end{array}\right.$ & $\begin{array}{l}\text { 0/0/0 (body, } \\
\text { spine, femur) }\end{array}$ & $\mid \begin{array}{c}1.9 \% / 7 \% / 5.4 \% \\
\text { (body, spine, femur) }\end{array}$ & & \\
\hline Storm, et al. [8] & Etidronate & Oral & $\begin{array}{c}54 / 100 \\
\text { patient years } \\
\text { (vertebral) }\end{array}$ & $\left\{\begin{array}{c}\text { 6/100 patient } \\
\text { years } \\
\text { (vertebral) }\end{array}\right.$ & & & & \\
\hline
\end{tabular}

Table 1: Study Results. 


\section{Journal of Orthopedics \& Bone Disorders}

\section{Discussion}

Overall, despite a few conflicting results, the study supports the hypothesis that bisphosphonate therapy in postmenopausal women with osteoporosis can prevent future fractures. The first type of data, based upon the randomized clinical trial literature, looked at rates of fractures in women who used bisphosphonate treatments and those who used placebo only. Five studies met all of the criteria to be included in this literature evaluation, and four of the five studies indicated unequivocally and at a statistically significant level that postmenopausal osteoporotic women who are given bisphosphonate treatment are less likely to have a future fracture than women who do not take the bisphosphonate treatment. However, one of the studies indicated that fracture rates post-treatment was significantly greater in the treatment group, rather than in the placebo group as reported by the other studies [7]. It is interesting to think about the Greenspan et al. study in light of a three year analysis of the bisphosphonate drugs ibandronate and risedronate among a large $(\mathrm{N}=1053)$ group of postmenopausal Japanese women [13]. That study found that among women who had increased bone mineral density after 6 months of taking either bisphosphonate had a reduced risk of fracture relative to that in women who also used the bisphosphonate treatment but did not have an increase in bone mineral density. It would be interesting to examine the Greenspan et al. data by stratifying the sample at certain bone mineral density gain parameters to see if there is a bone mineral density gain cutoff that can serve to predict fracture risk in osteoporotic postmenopausal women who have received bisphosphonate treatment.

Another outcome that was studied for this paper was bone mineral density in treatment and control groups. Because of the differences in the ways that these studies were conducted and reported, no further statistical analysis is possible for the present paper. However, each of these studies independently found that the bisphosphonates examined significantly increased the bone mineral density in the treatment groups relative to the placebo groups. All studies reviewed for this paper found that bone mineral density increased in the treatment groups relative to the control groups. If bone mineral density can be taken to indicate reduced risk of fracture, then these studies support the role of bisphosphonate therapy in postmenopausal osteoporotic women to prevent future fractures.

Concerning the third outcome, side effects, three studies did report adverse events/side effects, and these must be taken into consideration by any potential prescriber of bisphosphonates. Patient history and risk of fractures should be weighed against the risks associated with the potential adverse events.

\section{Critical Appraisals for Each Study}

Adami, et al. [10] was unclear about their randomization procedure, however the groups were similar to each other at the beginning of the study and there were no statistically significant differences between them. The groups were treated equally except for the intervention. Measures were objective and both patients and researchers were blind as to the grouping. The treatment effects were $4.7 \%$ and $6.1 \%$ for the 10 and 20 mg respective doses at the femoral neck, and 3.3\% and $3.8 \%$ at the trochanter, respectively. This study has a large sample size and is generalizable to all postmenopausal women. A criticism of this study is that it did not include an assessment of side effects.

Greenspan, et al. [7] used computerized randomization accomplished by the study biostatician. Groups were statistically similar to each other at the beginning of the study and groups were treated equally except for the intervention. Seventy-six percent of the enrolled participants completed the study. The treatment effects were measured in fracture rates and bone mineral density rates as reported above. One critique of this study is that although subjects in both groups were demographically similar to each other at baseline, the treatment group had more subjects with frailty; falls, diabetes and anticonvulsant use, and this could have biased the data to some extent.

Grey, et al. [11] used computerized randomization accomplished by the study statistician using a variable block size schedule. There were no demographic differences in the groups at the beginning of the study, and they were treated equally except for the intervention. Ninety-three percent of enrolled subjects completed the study. The treatment effects were measured in bone mineral density rates at $4.4 \%, 5.5 \%$ and $5.3 \%$ for $1 \mathrm{mg}, 2.5$ $\mathrm{mg}$, and $5 \mathrm{mg}$ zoledronate, respectively. The changes in bone mineral density in the hips were $2.6 \%, 4.4 \%$, and $4.7 \%$ for $1 \mathrm{mg}, 2.5 \mathrm{mg}$, and $5 \mathrm{mg}$ zoledronate, respectively. A critique of this study is that bone mineral density was used as a surrogate for fracture risk, and it would have been helpful to have fracture incidence reported as well.

Jacques, et al. [6] did not report their randomization procedures in this article. The study was double-blind and the groups were similar on demographic variables at the 


\section{Journal of Orthopedics \& Bone Disorders}

beginning of the study. The groups were treated equally except for the interventions. Because the study analyzed data from a different study, there were no participant drop-outs. The treatment effects were measured in bone mineral density rates and fracture rates as described above. This is a very large study and as such, the data are likely generalizable to all postmenopausal women with osteoporosis. A criticism of this study is that it did not include data on side effects.

Osaki, et al. [5] was a prospective matched cohort study and does not seem to have been randomized, nor was there blinding. The matching design made the subjects similar at the beginning of the study. Only fiftynine percent of the original subjects actually completed the study. Treatment effects were measured in fracture incidence over 36 months and were $4.3 \%$ in the treatment group compared to $13.1 \%$ in the control group, which was significant at $\mathrm{P}=0.10$. Because this is a cohort study, it is more difficult to ascertain cause and effect in terms of the efficacy of the treatment versus the control group.

Popp, et al. [12] did not describe the randomization procedures in this article. The study was double-blind and the groups were similar on demographic variables at the beginning of the protocol. The groups were treated equally except for the interventions. There were 107 patients included in the study and there appear to have been no losses to follow-up, but there were a few cases in which someone did not show up for a bone scan, according to the article. Treatment effects were measured in bone mineral density changes over 3 years and were a $9.58 \%$ increase in bone mineral density of the spine in the treatment group relative to the placebo group, and this change was significant at the $\mathrm{p}<0.0001$ level. The authors point out that although their study was large enough to compare the effects of the treatment versus the control; it was not large enough to establish the predictive value of the bone mineral density measurements in regard to fracture risk. Also, this study did not include an assessment of side effects, which is important to know for both the short- and long-term health of the women being treated.

Reid, et al. [9] used computerized randomization of their participants. The study was double blinded and the subjects were similar demographically at the beginning of the protocol. There were no reported drop-outs and 48 women participated. Treatment effects were reported in both bone mineral density changes and fracture rates, as described above. This is a moderate size study of 48 women, but it was continued for 2 years and the measurements of the bone mineral density were taken every 6 months, providing good estimates of change in BMD over that time period. A criticism of this study is that it did not include a report of side effects.

Storm, et al. [8] used computer-generation to randomize their patients. The study was double blinded and the groups were similar at the beginning of the protocol. Sixty-one percent of the enrolled participants completed the study. Treatment effects were measured as bone mass and fracture rates, as described above. This is a moderate size study of 66 women that was followed up for three years, and it is able to say that the bisphosphonate therapy increased bone mineral density in the women studied relative to the women who only had a placebo. A criticism is that this study did not include a report of side effects.

\section{Conclusions}

Postmenopausal women with osteoporosis who have suffered a fracture are at increased risk of future fractures due to loss of bone mineral density and deterioration of bone architecture. Bisphosphonates are drugs that can treat osteoporosis by inhibiting the resorption of bone, and thereby maintain or even increase bone mineral density and help to maintain the bone architecture. This review study searched the peer-reviewed scientific literature for clinical trials and/or systematic reviews and meta-analyses concerning the use of bisphosphonates to prevent future fractures in postmenopausal osteoporotic women. Four out of five studies found that women on bisphosphonate therapy were at significantly decreased risk of future fractures, although one smaller study found the opposite. Six studies found that bisphosphonate therapy significantly increased bone mineral density. The results indicate that bisphosphonate therapy can be used to increase bone mineral density and decrease the risk of future fractures, but some further research is warranted concerning the amount of bone mineral density increase necessary to reduce the risk of future fractures.

Two of the studies found independently of the other that fracture incidence were significantly less in the bisphosphonate treatment group than in the placebo control group, but the third found the opposite. Given that Hagino, et al. [13] found that among women who had increased bone mineral density after 6 months of taking either bisphosphonate had a reduced risk of fracture relative to that in women who also used the bisphosphonate treatment but did not have an increase in bone mineral density, it would be interesting to examine the Greenspan et al. data by stratifying the sample at 


\section{Journal of Orthopedics \& Bone Disorders}

certain bone mineral density gain parameters. This would allow the researchers to determine if there is a bone mineral density gain cutoff that can serve to predict fracture risk in osteoporotic postmenopausal women who have received bisphosphonate treatment.

\section{Recommendations}

Based upon the literature reviewed for this study, there is good evidence that bisphosphonate therapy increases bone mineral density and reduces the risk of fractures in post-menopausal women with osteoporosis. Both oral and I.V. treatments showed bone mineral density increases and, except for a single study, both types of administration of the therapy showed reduction in fracture risk over time. Future research on the best bisphosphonate with the optimal regimen for treatment of osteoporosis and prevention of fracture risk in postmenopausal women is called for. Additionally, although all studies did not report side effects, three of them did, and this was only over the period of time for a few years maximum. Further work on the risks and benefits of longterm bisphosphonate therapy would be advisable.

\section{Acknowledgment}

I would like to express my special thanks of gratitude to my teacher Branka Filipovic as well as my preceptor who gave me the golden opportunity to do this wonderful project on the topic "Bisphosphonates in Postmenopausal Women with Osteoporosis to Prevent Future Fractures". Secondly, I would also like to thank my parents and friends who helped me a lot in finalizing this project within the limited time frame.

\section{References}

1. Black DM, Rosen CJ (2016) Postmenopausal Osteoporosis. NEJM 374: 254-262.

2. Brown JP, Morin S, Leslie W, Papaioannou A, Cheung AM, et al. (2014) Bisphosphonates for Treatment of Osteoporosis: Expected Benefits, Potential Harms, and Drug Holidays. Can Fam Physician 60(4): 324333.

3. Kennel Kurt A, Matthew T Drake (2009) Adverse Effects of Bisphosphonates: Implications for Osteoporosis Management. Mayo Clin Proc 84(7): 632-638.

4. Donnelly E, Meredith DS, Nguyen JT, Gladnick BP, Rebolledo BJ, et al. (2012) Reduced Cortical Bone
Compositional Heterogeneity with Bisphosphonate Treatment in Postmenopausal Women with Intertrochanteric and Subtrochanteric Fractures. J Bone Miner Res 27(3): 672-678.

5. Osaki M, Tatsuki K, Hashikawa T, Norimatsu T, Chiba $\mathrm{K}$, et al. (2012) Beneficial Effect of Risedronate for Preventing Recurrent Hip Fracture in the Elderly Japanese Women. Osteoporosis International 23(2): 695-703.

6. Jacques, Richard M, Boonen S, Cosman F, Reid IR, et al. (2012) Relationship of Changes in Total Hip Bone Mineral Density to Vertebral and Nonvertebral Fracture Risk in Women with Postmenopausal Osteoporosis Treated with Once-Yearly Zoledronic Acid 5 Mg: The HORIZON-Pivotal Fracture Trial (PFT). J Bone Miner Res 27(8): 1627-1634.

7. Greenspan SL, Perera S, Ferchak MA, Nace DA, Resnick NM (2015) Efficacy and Safety of Single-Dose Zoledronic Acid for Osteoporosis in Frail Elderly Women. JAMA Internal Medicine 175(6): 913-921.

8. Storm T, Thamsborg G, Steiniche T, Genant HK, Sørensen OH (1990) Effect of Intermittent Cyclical Etidronate Therapy on Bone Mass and Fracture Rate in Women with Postmenopausal Osteoporosis. N Engl J Med 322(18): 1265-1271.

9. Reid IR, Wattie DJ, Evans MC, Gamble GD, Stapleton JP, et al. (1994) Continuous Therapy with Pamidronate, a Potent Bisphosphonate, in Postmenopausal Osteoporosis. The Journal of Clinical Endocrinology \& Metabolism 79(6): 1595-1599.

10. Adami S, Baroni MC, Broggini M, Carratelli L, Caruso I, et al. (1993) Treatment of Postmenopausal Osteoporosis with Continuous Daily Oral Alendronate in Comparison with Either Placebo or Intranasal Salmon Calcitonin. Osteoporos Int 3(S3): S21-S27.

11. Grey A, Bolland M, Mihov B, Wong S, Horne A, et al. (2014) Duration of Antiresorptive Effects of LowDose Zoledronate in Osteopenic Postmenopausal Women: A Randomized, Placebo-Controlled Trial. J Bone Miner Res 29(1): 166-172.

12. Popp AW, Guler S, Lamy O, Senn C, Buffat H, et al. (2013) Effects of Zoledronate versus Placebo on Spine Bone Mineral Density and Microarchitecture Assessed by the Trabecular Bone Score in Postmenopausal Women with Osteoporosis: A ThreeYear Study. J Bone Miner Res 28(3): 449-454. 


\section{Journal of Orthopedics \& Bone Disorders}

13. Hagino H, Yoshida S, Hashimoto J, Matsunaga M, Tobinai M, et al. (2014) Increased Bone Mineral Density with Monthly Intravenous Ibandronate Contributes to Fracture Risk Reduction in Patients with Primary Osteoporosis: Three-Year Analysis of the MOVER Study. Calcified Tissue International 95(6): 557-563. 